\title{
CD99 Is Strongly Expressed in Basal Cells of the Normal Adult Epidermis and Some Subpopulations of Appendages: Comparison with Developing Fetal Skin
}

\author{
Gawon Choi ${ }^{1,2 *} \cdot$ Jin Roh $^{3 *}$ \\ Chan-Sik Park ${ }^{3}$
}

${ }^{1}$ Graduate School of Medicine, University of Ulsan College of Medicine, Seoul; ${ }^{2}$ Mizmedi Hospital, Seoul; ; 3 Department of Pathology, Asan Medical Center, University of Ulsan College of Medicine, Seoul, Korea

\section{Received: March 23, 2016}

Revised: June 6, 2016

Accepted: June 19, 2016

\section{Corresponding Author}

Chan-Sik Park, MD, PhD

Department of Pathology, Asan Medical Center,

University of Ulsan College of Medicine, 88

Olympic-ro 43-gil, Songpa-gu, Seoul 05505, Korea

Tel: +82-2-3010-5838

Fax: $+82-2-472-7898$

E-mail: csikpark@amc.seoul.kr

${ }^{*}$ Gawon Choi and Jin Roh contributed equally to this work.

\begin{abstract}
Background: CD99 is a cell surface transmembrane glycoprotein expressed in various tissues. CD99 is differentially expressed between subpopulations of each tissue and is highly expressed in certain hematopoietic and precursor cells. However, there has been no comprehensive study of CD99 expression in normal skin. We evaluated CD99 expression in normal human skin and developing fetal skin. Methods: Seventy-five adult skin samples containing normal skin and eight fetal skin samples of different gestational ages were collected. CD99 immunohistochemical staining was performed to evaluate expression pattern in adult and fetal skin samples. CD99 and CD34 expression were compared by double immunofluorescence. Results: In normal adult skin, CD99 was strongly expressed in the membrane of epidermal basal keratinocytes, hair follicle bulges and outer root sheaths, and inner secretory cells of eccrine sweat glands. In fetal skin, CD99 was not expressed on the periderm at 16 weeks of gestation but was expressed in basal cells of fetal skin at around 19 weeks of gestation. CD99 expression became comparable to that of the adult skin after 20 weeks of gestation. CD99 and CD34 were co-expressed in hair follicle outer root sheaths, as seen by double immunofluorescence study. Conclusions: This is the first study examining CD99 expression pattern in normal adult and fetal skin. CD99 tends to be expressed in the basal/precursor cells of epidermis and in hair follicles. These results provide a basis for future investigation on functions of CD99 in the skin and provide a novel potential target for the treatment of dermatologic lesions.
\end{abstract}

Key Words: CD99 protein; Skin; Immunohistochemistry
CD99 is a 32-kD type I transmembrane glycoprotein encoded by the $C D 99$ gene and its expression has been reported in many cell types, such as hematopoietic cells, endothelial cells, central nervous system ependymal cells, thymocytes, granular cells of the ovary, Sertoli cells, and pancreatic islet cells. ${ }^{1-3} \mathrm{CD} 99$ expression is seen in all leukocyte lineages but is differentially expressed with maturation and is particularly strong in immature thymic T-lineage cells. ${ }^{2}$ Other studies also demonstrated high CD99 expression in tonsillar lymphoid progenitor cells ${ }^{4}$ and in the subventricular zone of fetal brain (C.-S. Park, unpublished data), suggesting that $\mathrm{CD} 99$ may play important roles in these precursor cells. The functions of CD99 in cells in which CD99 was highly expressed have been studied and they were as follows: cell death of thymocytes and T lymphocytes, ${ }^{5,6}$ migration through monocyte endothelial junctions by adhesion and diapedesis, ${ }^{7}$ cell-cell adhesion in lymphocytes, ${ }^{5,8}$ maintenance of cellular morphology in Hodgkin and Reed/Sternberg cells, ${ }^{9}$ and recruitment of T cells to inflamed mouse skin. ${ }^{10}$ Pathologically, CD99 is strongly expressed in various tumors including Ewing sarcoma/ peripheral neuroectodermal tumors, T-lymphoblastic lymphoma, and others. ${ }^{11}$ CD99 is a crucial marker for the diagnosis of these tumors.

Skin is the largest human organ and is structurally composed of epidermis, dermis, and appendages including hair follicles, sebaceous glands, and sweat glands (eccrine and apocrine). Microscopically, cells which composing the skin showed various stages of differentiation. The epidermis contains multiple layers of stratified keratinocytes, and basally located cells are morphologically undifferentiated. Hair follicles are cylindrical invaginations of surface epithelium, and are composed of the innermost hair shaft, the inner root sheath (IRS), and the outer root sheath (ORS). The bulge, the insertion site of the arrector pilli muscle, is the prominent epithelial protuberance of the ORS and it contains basaloid cells. ${ }^{12}$ Sebaceous glands have central acini, mass- 
es of rounded cells that are packed with lipid-filled vacuoles and peripheral undifferentiated basal cells. Eccrine sweat glands consist of a secretory portion lined with stratified cuboidal cells and an excretory portion lined with two layers of smaller cuboidal cells. Apocrine glands are large glands and secretory cells usually show cuboidal morphology with eosinophilic cytoplasm. ${ }^{13}$

CD99 expression levels differ in specific subpopulations according to cellular maturation. We identified strong CD99 expression in the basally located precursor cells of normal epidermis. We inferred that CD99 shows differential expression between the epidermis and appendageal subpopulations with maturation. No systemic evaluation of CD99 expression in normal skin or the appendages has yet been published. The aim of the present study was to evaluate CD99 expression patterns in normal adult epidermis and appendages by immunohistochemistry (IHC) and to ascertain CD99 expression in normal fetal skin during different gestational ages. We also investigated whether morphologically immature subpopulations that express CD99 correlate with skin precursor cells based on previously established marker.

\section{MATERIALS AND METHODS}

\section{Skin samples}

In this study, we retrospectively selected 75 cases of formalinfixed, paraffin-embedded hair-bearing adult skin samples between 2002 and 2009 at Asan Medical Center. Samples were taken from the margins of routine surgical procedures such as excisional biopsy or excision for benign/malignant skin lesions that were diagnosed as following: various inflammatory lesions with parakeratosis $(n=16)$, keratinocytic tumors $(n=11)$, benign tumors with apocrine and eccrine differentiation $(\mathrm{n}=14)$, malignant tumors with follicular differentiation $(\mathrm{n}=1)$, benign tumors with follicular differentiation ( $\mathrm{n}=17)$, and tumors with sebaceous differentiation $(n=12)$. The rest were retrieved from mature cystic teratoma $(\mathrm{n}=2)$ and normal scalp tissues biopsied for therapeutic reasons $(n=2)$. To analyze CD99 expression patterns in fetal skin, eight fetal skin samples were collected from the archives of Asan Medical Center. Gestational ages of the collected cases were 16, 17, 19 (2 cases), 20, 25, 26, and 28 weeks. All tissues were immediately fixed in $10 \%$ buffered formalin, and then processed in paraffin wax using standard procedures. Sections were serially cut into $4-\mu \mathrm{m}$ sections and stained with hematoxylin and eosin for examination. The study protocol was approved by the Institutional Review Board (project number 2009-440) of Asan Medical Center.

\section{Immunohistochemical staining}

All hematoxylin and eosin-stained slides were reviewed to ensure quality. When multiple blocks were available in a single case, one block containing representative tissue was selected. IHC staining for CD99 was performed using a BenchMark XT autoimmunostianer (Ventana Medical Systems, Tucson, AZ, USA) according to the manufacturer's instructions and using the reagents supplied with the kit. In brief, 4- $\mu \mathrm{m}$ sections were mounted on silanized charged slides, dried for 10 minutes at room temperature and then further dried for 20 minutes at $65^{\circ} \mathrm{C}$. After deparaffinization, heat-induced epitope retrieval using standard Cell Conditioning Solution 1 was performed for 24 minutes. Subsequently, primary anti-CD99 (1:100, clone DN16, DiNonA, Seoul, Korea) was applied by an automated immunostaining system with the UltraView DAB Detection Kit (Ventana Medical Systems). Immunostained sections were counterstained with hematoxylin. Analysis of whole tissue section slides stained for $\mathrm{CD} 99$ by IHC was performed under a light microscope as previously described. ${ }^{14-16}$ Lymphocytes and endothelial cells were used as internal positive controls. The results of CD99 immunostaining were classified into the following groups: consistently complete and strong membrane staining was assigned a value ++ , weak to moderate membrane staining was assigned a value of + , variable membrane staining was assigned a value of $+/-$, and no staining was assigned a value of -. Occasional cytoplasmic staining was minimal and weak.

\section{Immunofluorescence assay}

For immunofluorescence (IF) staining, 4- $\mu \mathrm{m}$ sections were incubated with mouse anti-CD99 (1:200, clone DN16, DiNo$\mathrm{nA})$ and rabbit anti-CD34 (1:10, \#ab64480, rabbit polyclonal, Abcam, Cambridge, UK) for 60 minutes at room temperature. Sections were then incubated with tetramethylrhodamine-conjugated host anti-mouse IgG antibody and fluorescein isothiocyanate-conjugated host anti-rabbit IgG antibody for 60 minutes at room temperature. Nuclei were stained with DAPI. Sections incubated with the appropriate isotype control primary antibodies and fluorescently labeled secondary antibodies were utilized as necessary. Results were analyzed using confocal microscopy (Leica TCS_NP/SP, Leica Microsystems, Mannheim, Germany).

\section{RESULTS}

\section{Basal cells showed strong CD99 expression in normal adult epidermis}

To examine the expression pattern of CD99 in epidermal ke- 
ratinocytes, immunostaining for CD99 was performed on nonpathologic adult skin samples (Fig. 1A). In the epidermis, cell membranes in the basal cell layer were strongly stained (Fig. 1B), whereas the keratinocytes in the prickle cell layer just above the basal cell layer showed markedly reduced staining and cells in the granular and hornified layers did not express CD99. We also identified intense CD99 expression in Langerhans' cells in the epidermal layer (Fig. 1B).

\section{Basaloid cells of bulge and ORS showed strong CD99 expression in normal adult hair follicle}

Infundibula of hair follicles possessed a CD99 expression pattern similar to that of the epidermis. CD99 staining was strong in the basaloid cells (Fig. 1C) at the bulges, and basal cells of the follicular ORS were also strongly stained (Fig. 1D). Other subpopulations of hair follicles showed varying immunoreactivity. When observing CD99 staining in the central shaft of hair follicles, the cuticle was negative for CD99, the lower IRS was weakly positive, and the ORS was strongly positive (Fig. 1D).

\section{CD99 was differentially expressed in subpopulations of normal adult sweat glands and sebaceous glands}

Secretory apocrine glands showed variable CD99 expression. Relatively thin to flat cell-lined apocrine glands were negative for CD99, but relatively tall cell-lined apocrine glands were positive for CD99. The apical luminal surfaces of both flat and tall
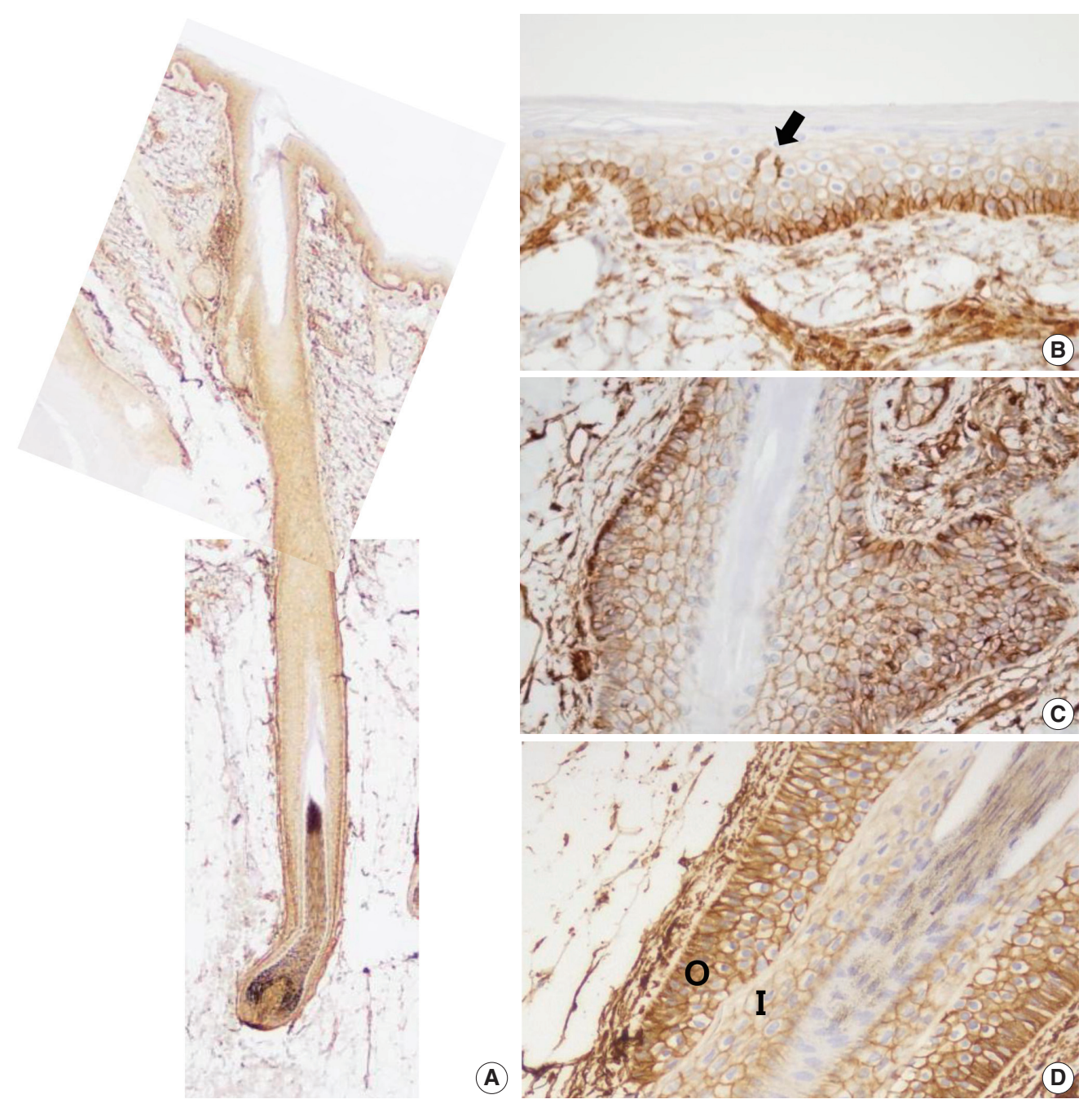

Fig. 1. CD99 expression in normal adult epidermis and hair follicles. (A) Overall CD99 expression pattern in normal adult skin. (B) Basal cells of the epidermis show strong immunopositivity for CD99. Epidermal Langerhans cells are also strongly immunopositive for CD99 (arrow). (C) Basaloid cells in the bulge, the insertion of the arrector pili muscle, show high CD99 expression. (D) In a terminal anagen hair follicle, the outer root sheath cells are strongly positive for CD99 stain. The inner root sheath cells are weakly positive for CD99 stain. O, outer root sheath; I, inner root sheath. 


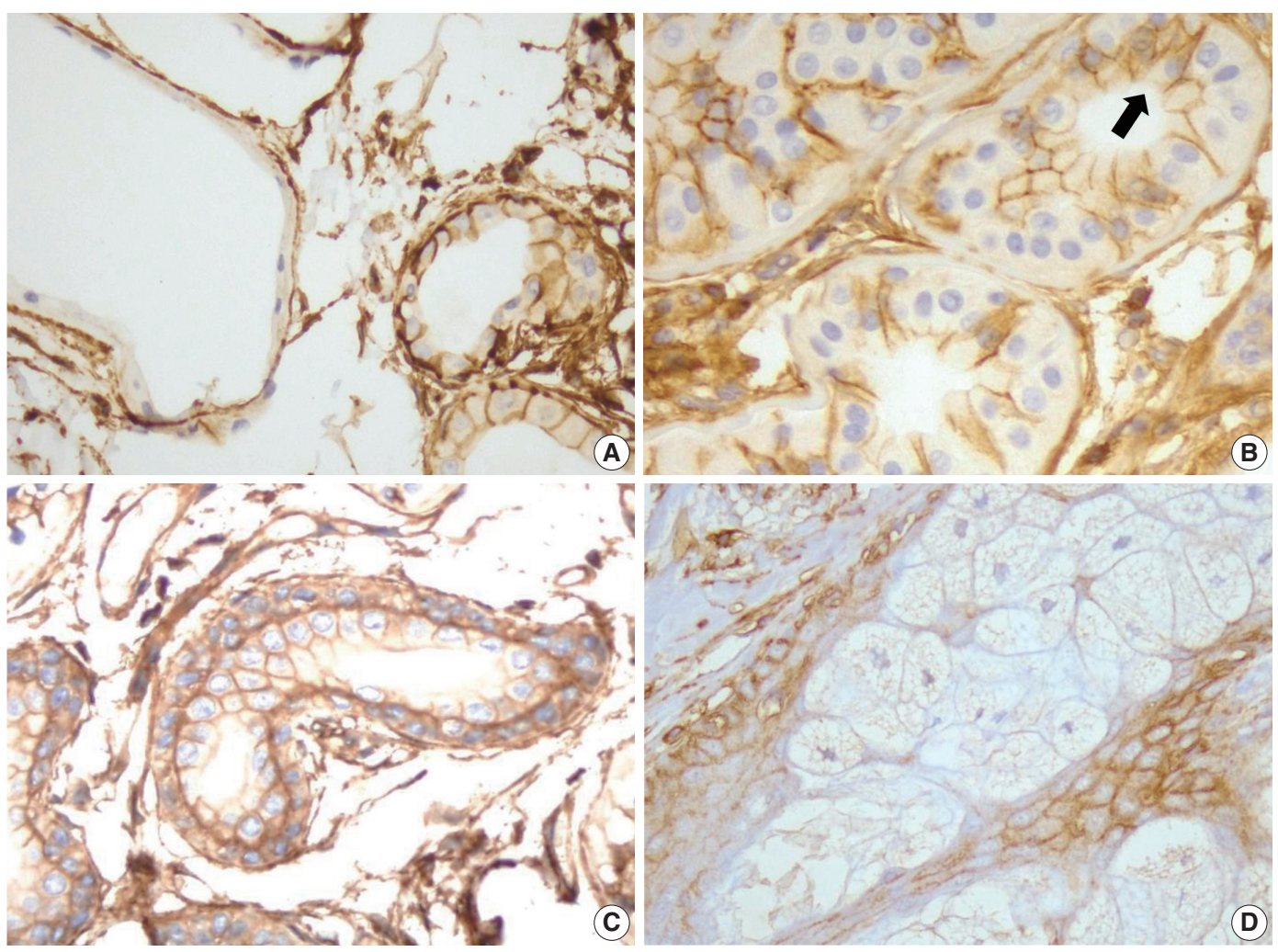

Fig. 2. CD99 expression in the normal adult sweat glands and sebaceous glands. (A) In apocrine glands, flattened lining cells are immunonegative for CD99 (left). Meanwhile, tall cells in apocrine glands express CD99 (right). (B) Inner small cells of eccrine glands express CD99 in opposition to outer large cells (arrow). Apicoluminal surface of the eccrine glands are immunonegative for CD99. (C) Two layers of excretory ductal cells are immunopositive for CD99. (D) In sebaceous glands, outer basal cells express CD99. However, mature sebocytes do not express CD99.

cell-lined glands were CD99-negative (Fig. 2A). Secretory coils of eccrine sweat glands are composed of outer clear cells and inner cuboidal cells; the outer clear cells were immunonegative and scattered inner cuboidal cells were immunopositive for CD99 (Fig. 2B). The excretory duct is composed of two-layered cuboidal cells, and both layers were immunopositive for CD99 (Fig. 2C). The apical luminal surfaces of inner eccrine cells and inner excretory ducts also lacked CD99 expression (Fig. 2B, C). In the sebaceous glands, basal cells surrounding the sebaceous alveolus were CD99 immunopositive, but mature sebocytes containing central lipid droplets were immunonegative for CD99 (Fig. 2D). Cells that were positive for CD99 immunostaining in normal adult epidermis and skin appendages are summarized in Table 1.

\section{CD99-positive epidermal basal cells appeared in fetal skin early in the second trimester}

Eight developing fetal human skin samples were obtained from aborted fetuses of 16-28 gestational weeks. In early stages, fetal skin is called periderm and is composed of $2-3$ epider-
Table 1. Summary of CD99 expression in normal adult epidermis and skin appendages

\begin{tabular}{llc}
\hline & & Immunoreactivity \\
\hline Epidermis & Basal cells & ++ \\
& Prickle cells & + \\
& Granular cells & - \\
& Horny cells (cornified cells) & - \\
Hair follicle & Bulge & ++ \\
& Cortex & - \\
& Cuticle & - \\
& Inner root sheath-lower & + \\
Sweat glands & Outer root sheath & ++ \\
Eccrine secretory coil & Glassy membrane & - \\
& & \\
Apocrine secretory acinus & Inner dark cells & Secretory cells \\
Excretory duct & Inner layer cells & $++^{\mathrm{a}}$ \\
& Outer layer cells & + \\
Sebaceous glands & Mature sebocytes & $+^{\mathrm{a}}$ \\
& Basal cells & ++ \\
\hline
\end{tabular}

++ , consistently strong and complete membranous staining; +, consistently weak to moderate staining; +/-, variable staining; -, negative.

${ }^{a}$ Apico-luminal surfaces are negative for CD99. 

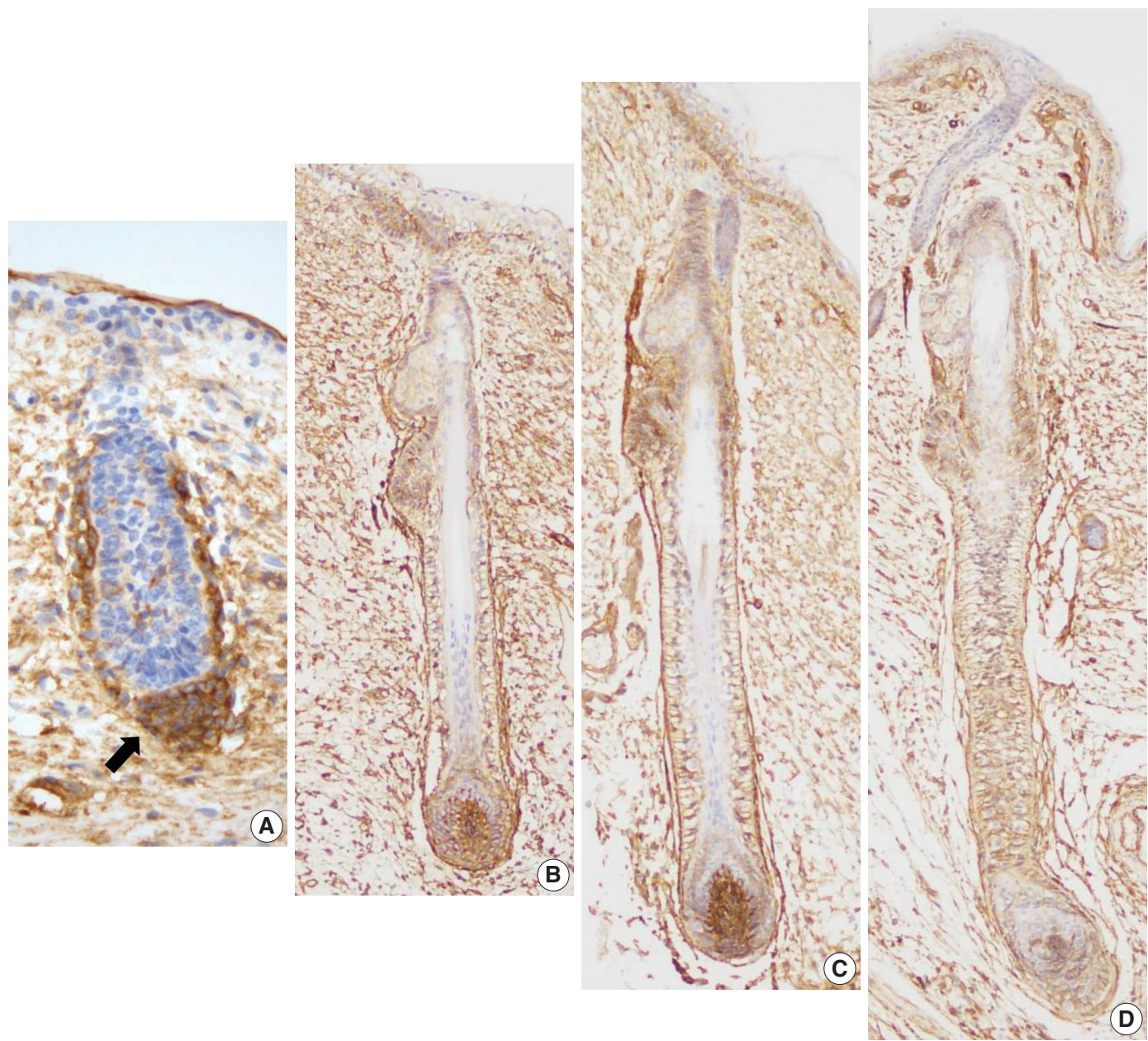

Fig. 3. CD99 expression pattern during normal development of the hair follicles. (A) At 16 weeks of gestation, only mesenchymal cells around the hair germ express CD99 (arrow). (B) CD99 positive epidermal basal cells appear at 19 weeks of gestation. Basaloid cells in the bulge and basal cells of the outer root sheath and lower inner root sheath also express CD99. (C, D) CD99 expression pattern is identical to that of adult skins at 20 and 25 weeks of gestation.

mal cell layers. ${ }^{17}$ At 16 weeks of gestation, the periderm, including the epidermal basal layer and hair follicle germ, was negative for CD99, whereas mesenchymal cells around the hair germ were strongly positive for CD99 (Fig. 3A). At 19 weeks of gestation, the fetal epidermis and skin appendages start to resemble normal adult skin; basal cell layer, intermediate cell layer, hair follicles, sweat glands, and sebaceous glands appear at this stage. CD99 was expressed in the epidermal basal cell layer, hair bulge, ORS, and dermal papilla (Fig. 3B). At 20 and 25 weeks of gestation, fetal skin is histologically similar to adult skin, showing CD99 immunoreactivity patterns identical to those of adult skins (Fig. 3C, D).

CD99 and CD34 were co-expressed in lower ORS cells, but they did not co-localize

In our study, CD99 staining was particularly strongly positive in immature epidermal basal cells and cells at the bulge. We therefore compared CD99-positive subpopulations of normal epidermis and skin appendages to CD34-positive subpopulations that have been reported as cutaneous precursor cells using double-IF. We focused on expression patterns of these markers in the basal cells of epidermis and hair follicles. In the adult epidermis and skin appendages, CD99 and CD34 expression partially overlapped at the lower ORS (Fig. 4A, D). Epidermal basal cells and basaloid cells of the bulge were CD34-negative (Fig. 4A-C). In the 20-week-old fetal skin, CD99 and CD34 were not co-expressed in the basal cells of epidermis (Fig. 5B), bulge (Fig. 5C), or ORS; however, mesencymal cells of dermal papilla showed co-expression (Fig. 5A). 


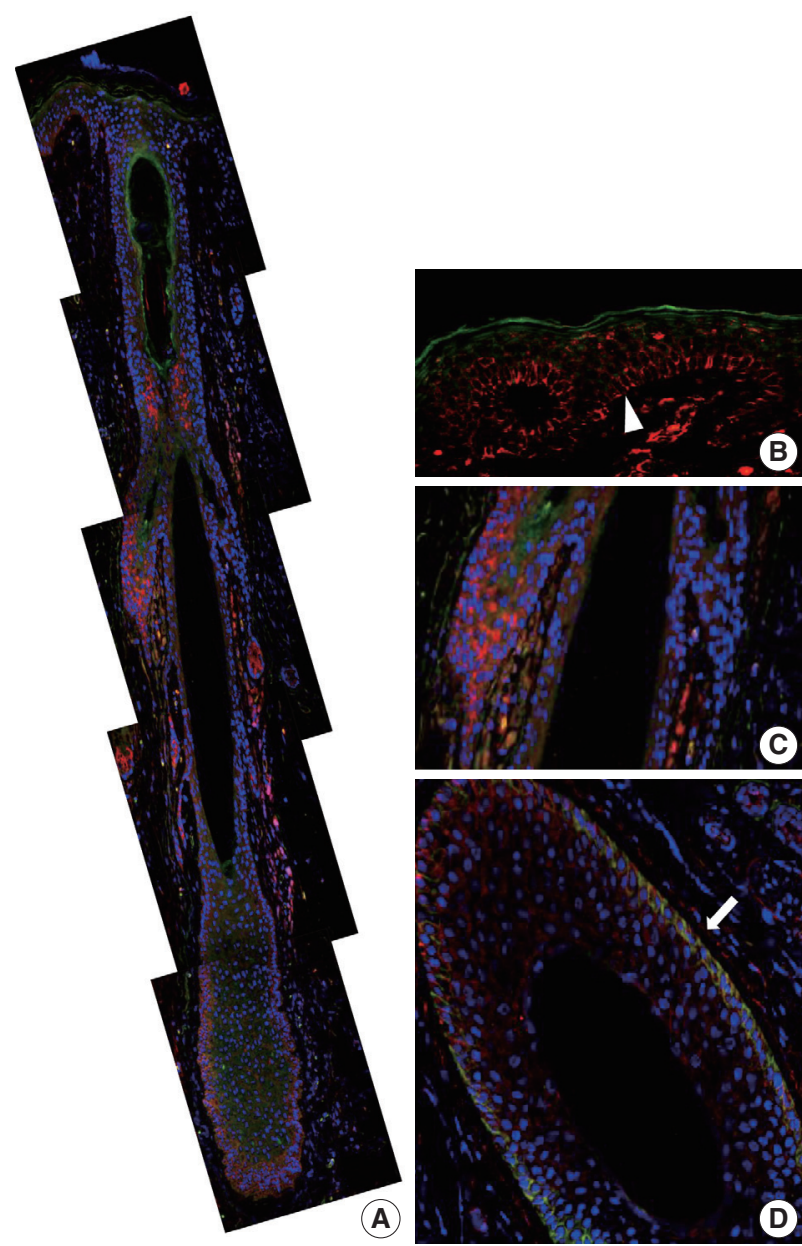

Fig. 4. CD99 and CD34 expression pattern by double-immunofluorescence staining in normal adult epidermis and hair follicles. (A) Overall expression pattern of CD99 (red) and CD34 (green). Epidermal basal cells (arrowhead) $(B)$ and basaloid cells $(C)$ in the bulge only express CD99 but not CD34. (D) Some cells in the lower outer root sheath show co-expression of CD99 and CD34 (arrow) (horizontal section).

\section{DISCUSSION}

The aim of the current study was to examine CD99 expression in normal human skin and skin appendages; no such study has been performed to date. Differential expression of CD99 was observed in the epidermis and appendages. We demonstrated that CD99 was strongly expressed in the immature epidermal basal cells, but the fully differentiated granular layer did not show CD99 positivity in normal adult skin. This result is consistent with the findings of a previous study ${ }^{18}$ in which CD99 expression was evaluated in a large series of cutaneous melanoma samples and was described briefly as a crisp membranous staining on normal epidermal basal cells. Our study showed that in skin appendages, CD99 showed differential immunoreactivity

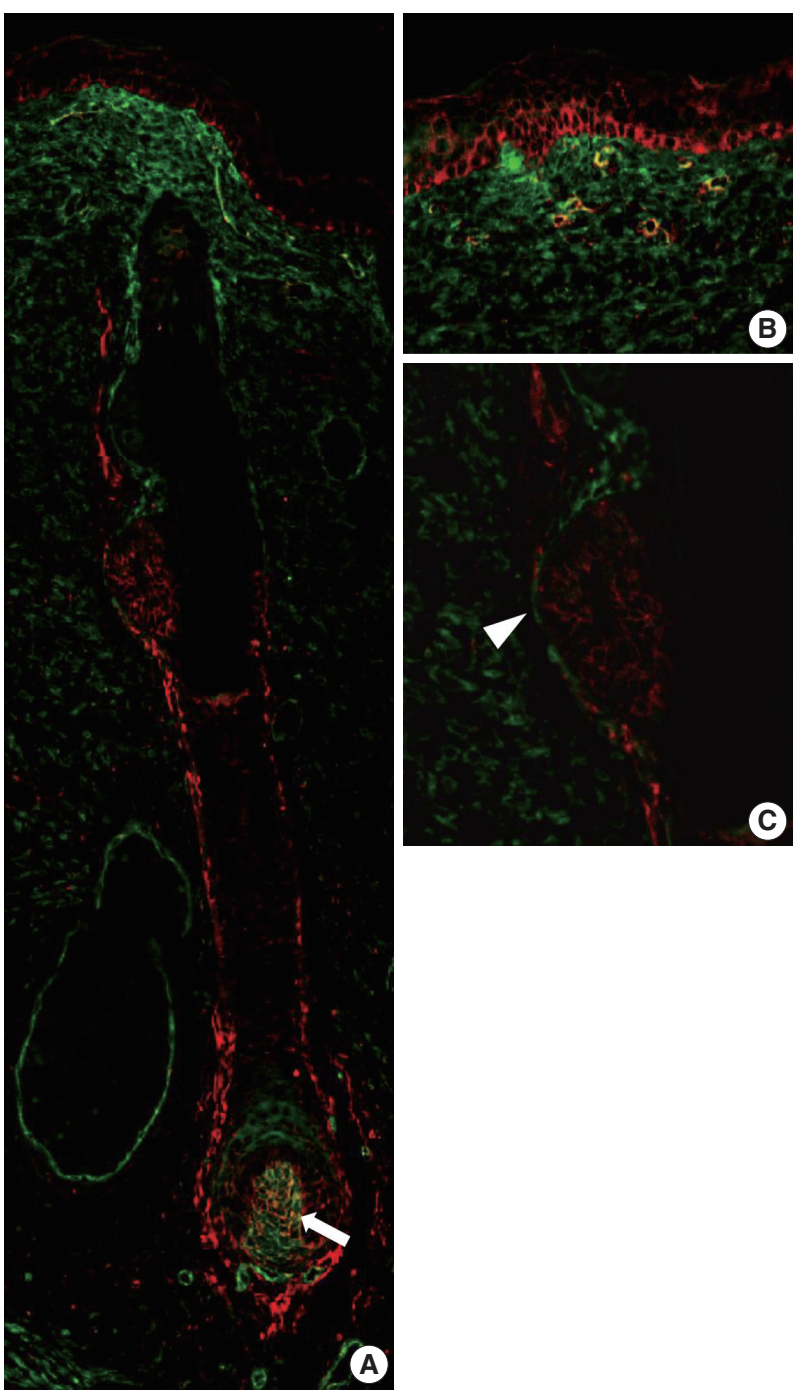

Fig. 5. CD99 and CD34 expression pattern by double-immunofluorescence staining in the fetal epidermis and hair follicles at 20 weeks of gestation. (A) Overall expression pattern of CD99 (red) and CD34 (green). Dermal papilla expresses both CD99 (red) and CD34 (green) (arrow). (B, C) Epidermal basal cells and basaloid cells in the bulge (arrowhead) express only CD99.

in each subpopulation. Bulge cells, basal cells of the ORS, and eccrine inner cuboidal cells were consistently and strongly positive. However, CD99 expression was variably positive in apocrine secretory glands. Relatively tall cells with luminal decapitation tended to be CD99-positive, but thin to flat cells without decapitation tended to be negative. These findings suggest that CD99 expression can differ according to cellular functional status.

Analysis of CD99 expression was also performed during different developmental stages of fetal skin. In fetal skin, peridermal cells persist until about week 21 and gradually disappear as stratum corneum. In the early stages of epidermal development, 
mitotic activity occurs in all the epidermal layers; however, when differentiation begins, mitotic activity becomes restricted to the cells of the basal layer. ${ }^{17}$ Angiotensin converting enzyme (ACE), keratin 19, $\beta 1$-integrin, and p63 were used as stem cell markers in examining the various developmental stages of fetal skin by double IF. ${ }^{19}$ The expression of ACE, $\beta 1$-integrin, keratin 19 , and p63 was seen in all epidermal layers of the developing fetal skin at 11-20 weeks of gestation. From 21 weeks of gestation onward, their expression was mainly confined to the basal layer of epidermal cells. In our study, CD99 was not seen in the periderm at 16 weeks of gestation but was consistently expressed at 19 weeks of gestation. Based on this result, we suggest that CD99 expression precedes the expression of ACE, $\beta 1$-integrin, keratin 19 , and p63 in the fetal basal layer.

Based on the particularly strong CD99 expression in immature thymic T-lineage cells, ${ }^{2}$ tonsillar lymphoid progenitor cells, ${ }^{4}$ and subventricular zone of fetal brain (C.-S. Park, unpublished data), we attempted to correlate CD99 expression with immature subpopulations of epidermis and appendages. CD99 was strongly expressed in immature basal keratinocytes. In hair follicles, the bulge is a prominent epithelial protuberance of the ORS. There is evidence for populations of stem cells located at the basal layer of the epidermis and the hair follicle bulge. CD99 is also strongly expressed in the bulge.

According to previous reports that analyzed expression patterns of various precursor markers, $\mathrm{CD} 34$ is more specific for the peripheral layer of the ORS, cytokeratin (CK) 15 for bulge and epidermal basal cells, nestin for inner portions of the ORS, CK19 for basal keratinocytes in the upper and lower third of the ORS, p63 for the basal/suprabasal layer, hair matrix, and ORS, and CD200 for the bulge. ${ }^{20-24}$ We found CD99 was more broadly expressed in the epidermis and appendages. We showed that CD34, which was thought to have similar expressions patterns to CD99, shows different expression by double IF. In the present study, CD34 and CD99 were only co-expressed in the lower ORS. Therefore, we assumed that precursor cell subpopulations in the skin are more varied than previously thought.

In conclusion, this study examined CD99 expression in normal adult and fetal skin. CD99 is strongly expressed in the immature epidermal basal cells in the bulge and basal cells of the hair follicle ORS. These findings suggest that CD99 may carry out a specific function in the structures where it is expressed. Interestingly, CD99-expressing cell subpopulations partially overlapped with sites harboring epidermal precursor cells. Therefore, we present that $\mathrm{CD} 99$ is a unique and previously unreported marker of epidermis and its appendages. Future studies should focus on functions of CD99 in the epidermis and its appendages and investigate its feasibility as a novel target for the treatment of dermatologic lesions.

\section{Conflicts of Interest}

No potential conflict of interest relevant to this article was reported.

\section{Acknowledgments}

This work was supported by the National Research Foundation of Korea, Grant No. 2008-0062286 (http://www.nrf. re.kr/) and the Asan Institute for Life Sciences, Grant No. 2012527 (http://en.ails. amc.seoul.kr/).

\section{REFERENCES}

1. Aussel C, Bernard G, Breittmayer JP, Pelassy C, Zoccola D, Bernard A. Monoclonal antibodies directed against the E2 protein (MIC2 gene product) induce exposure of phosphatidylserine at the thymocyte cell surface. Biochemistry 1993; 32: 10096-101.

2. Dworzak MN, Fritsch G, Buchinger P, et al. Flow cytometric assessment of human MIC2 expression in bone marrow, thymus, and peripheral blood. Blood 1994; 83: 415-25.

3. Gordon MD, Corless C, Renshaw AA, Beckstead J. CD99, keratin, and vimentin staining of sex cord-stromal tumors, normal ovary, and testis. Mod Pathol 1998; 11: 769-73.

4. Strauchen JA, Miller LK. Lymphoid progenitor cells in human tonsils. Int J Surg Pathol 2003; 11: 21-4.

5. Bernard G, Zoccola D, Deckert M, Breittmayer JP, Aussel C, Bernard A. The E2 molecule (CD99) specifically triggers homotypic aggregation of CD4+ CD8+ thymocytes. J Immunol 1995; 154: 26-32.

6. Pettersen RD, Bernard G, Olafsen MK, Pourtein M, Lie SO. CD99 signals caspase-independent $\mathrm{T}$ cell death. J Immunol 2001; 166: 4931-42.

7. Schenkel AR, Mamdouh Z, Chen X, Liebman RM, Muller WA. CD99 plays a major role in the migration of monocytes through endothelial junctions. Nat Immunol 2002; 3: 143-50.

8. Hahn JH, Kim MK, Choi EY, et al. CD99 (MIC2) regulates the LFA1/ICAM-1-mediated adhesion of lymphocytes, and its gene encodes both positive and negative regulators of cellular adhesion. J Immunol 1997; 159: 2250-8.

9. Kim SH, Choi EY, Shin YK, et al. Generation of cells with Hodgkin's and Reed-Sternberg phenotype through downregulation of CD99 (Mic2). Blood 1998; 92: 4287-95.

10. Bixel G, Kloep S, Butz S, Petri B, Engelhardt B, Vestweber D. Mouse 
CD99 participates in T-cell recruitment into inflamed skin. Blood 2004; 104: 3205-13.

11. Ambros IM, Ambros PF, Strehl S, Kovar H, Gadner H, SalzerKuntschik M. MIC2 is a specific marker for Ewing's sarcoma and peripheral primitive neuroectodermal tumors: evidence for a common histogenesis of Ewing's sarcoma and peripheral primitive neuroectodermal tumors from MIC2 expression and specific chromosome aberration. Cancer 1991; 67: 1886-93.

12. Narisawa $Y$, Kohda H. Two- and three-dimensional demonstrations of morphological alterations of early anagen hair follicle with special reference to the bulge area. Arch Dermatol Res 1996; 288: 98-102.

13. Young B, Lowe JS, Stevens A, Heath JW. Wheater's functional histology: a text and colour atlas. 5th ed. Philadelphia: Elsevier, 2006; 167-85.

14. Park CK, Shin YK, Kim TJ, Park SH, Ahn GH. High CD99 expression in memory $\mathrm{T}$ and $\mathrm{B}$ cells in reactive lymph nodes. J Korean Med Sci 1999; 14: 600-6.

15. Cho EY, Choi Y, Chae SW, Sohn JH, Ahn GH. Immunohistochemical study of the expression of adhesion molecules in ovarian serous neoplasms. Pathol Int 2006; 56: 62-70.

16. Shin SJ, Lee H, Jung G, et al. Expression of CD99 in multiple myeloma: a clinicopathologic and immunohistochemical study of 170 cases. Korean J Pathol 2014; 48: 209-16.
17. Holbrook KA. Structure and function of the developing human skin. In: Goldsmith LA, ed. Biochemistry and physiology of the skin. New York: Oxford University Press, 1983; 64-101.

18. Wilkerson AE, Glasgow MA, Hiatt KM. Immunoreactivity of CD99 in invasive malignant melanoma. J Cutan Pathol 2006; 33: 663-6.

19. Liu HW, Cheng B, Li JF, et al. Characterization of angiotensin-converting enzyme expression during epidermis morphogenesis in humans: a potential marker for epidermal stem cells. Br J Dermatol 2009; 160: 250-8

20. Jiang S, Zhao L, Purandare B, Hantash BM. Differential expression of stem cell markers in human follicular bulge and interfollicular epidermal compartments. Histochem Cell Biol 2010; 133: 455-65.

21. Inoue $\mathrm{K}$, Aoi N, Sato T, et al. Differential expression of stem-cell-associated markers in human hair follicle epithelial cells. Lab Invest 2009; 89: 844-56.

22. Hoang MP, Keady M, Mahalingam M. Stem cell markers (cytokeratin 15, CD34 and nestin) in primary scarring and nonscarring alopecia. Br J Dermatol 2009; 160: 609-15.

23. Gho CG, Braun JE, Tilli CM, Neumann HA, Ramaekers FC. Human follicular stem cells: their presence in plucked hair and follicular cell culture. Br J Dermatol 2004; 150: 860-8.

24. Pellegrini G, Dellambra E, Golisano O, et al. p63 identifies keratinocyte stem cells. Proc Natl Acad Sci U S A 2001; 98: 3156-61. 